\title{
Internet of Things (IoT) Based Smart Home Automation and Scrutiny System
}

\author{
Mr. Nerella Ome, Master of Engineering, Assistant Professor, GRIET- Hyderabad, Telangana, \\ India. omenerella@gmail.com
}

Mr. Someswara Rao Gaddala, M.Tech, Assistant Professor, VJIT-Hyderabad, Telangana, India. someshg.somu@gmail.com

\begin{abstract}
Internet of Things (IoT) is a developing innovation that makes our reality more intelligent. Now a days, the home security is fundamental as a potential results of interference are growing well ordered. Security has turned into a significant issue everywhere. Using IoT based Smart Home Automation and Scrutiny System , we can monitor home environmental parameters ( such as Temperature, Gas detection, Light Intensity, Humidity, Fire, Motion and Image) and control the appliances . It can identify the people who are in the home, with the assistance of the PIR sensor and camera. If Data is abnormal then authorized persons get notification via e-mail. By using android based apps we can monitor home environmental parameters and control the home appliances. The focus of this paper is to give preferable security system over the present level of security in the home. This system is appropriate for ongoing home security checking and for remotely controlling the home appliances.
\end{abstract}

Keywords - Arduino IDE, DHT11 sensor, ESP8266,Internet of Things (IoT), NodeMCU, Thingspeak.

\section{INTRODUCTION}

The Internet of Things (IoT), which is an advanced and efficient solution for connecting the things to the internet and to connect the entire world of things in a network. Internet of Things where 'things' like sensors and devices transmit data directly to the Internet has become an enabling technology eco-system with several applications. Smart Home, Smart Farming, Smart Grid, Smart Health, Smart Supply Chain etc., are some of the applications of IoT in different fields. The IoT Technology involves sensors, Microcontrollers, Wireless networking, Cloud based services, Mobile apps and Web pages.

Observing facilities will be essential and helpful for our day by day life, since it is significant for us to consider our security. This work created a system, which is sorted out with an incorporated web server, exceptionally verified cameras, Wi-Fi gadgets are associated with the web. A particular server is situated in a focal point of our project, which is called Integrated Server, which intermittently getting the recordings from some surveillance cameras through the private system. Such recordings are transmitted from the cameras to the server. The Integrated Server requires organize cameras to transmit video at an examining rate and packs the video to MPEG at that point aggregates a progression of them in the capacity. These system catches data and transmits the live video streams by means of Wi-Fi remote handset for IoT module to a Smart telephone individual application by utilizing the web.

\section{PROPOSED SYSTEM}

An IoT Eco System consists of the following

1. An IoT Hardware Platform 2. Web Services

3. User Interfaces 4. Data Analytics.

The main objectives of this research proposal are To Design and Development of an IoT based Eco System. To Develop Web based services for IoT Platform. To Develop Microcontroller programming for IoT application. To Develop Android /Mobile Applications for IoT.

An IoT Hardware Platform: It consists of Microcontroller, Sensors, Wi-Fi module and Actuators.

Development Boards: There are many Development boards to read the sensors data and process it for various user defined applications. The Arduino Uno, NodeMCU, Photon, Raspberry Pi and MSP430 launch boards are happening boards with ease of coding, availability and with economic feasibility. The Node MCU is the selection of choice for developing an IoT Eco system because of its robust performance, on board $\mathrm{WiFi}$ module, durability and easiness of programming for the IoT applications.

The system consists of NodeMCU as a main processing unit for the entire system and all the sensors (DHT11, MQ2, KY-026, HC-SR501, LDR) and devices can be connected with the NodeMCU. To the NodeMCU the sensors are connected \& operated by it and it is used to retrieve the data and processes the analysis with the sensor data and updates it to the cloud through a Wi-Fi . 


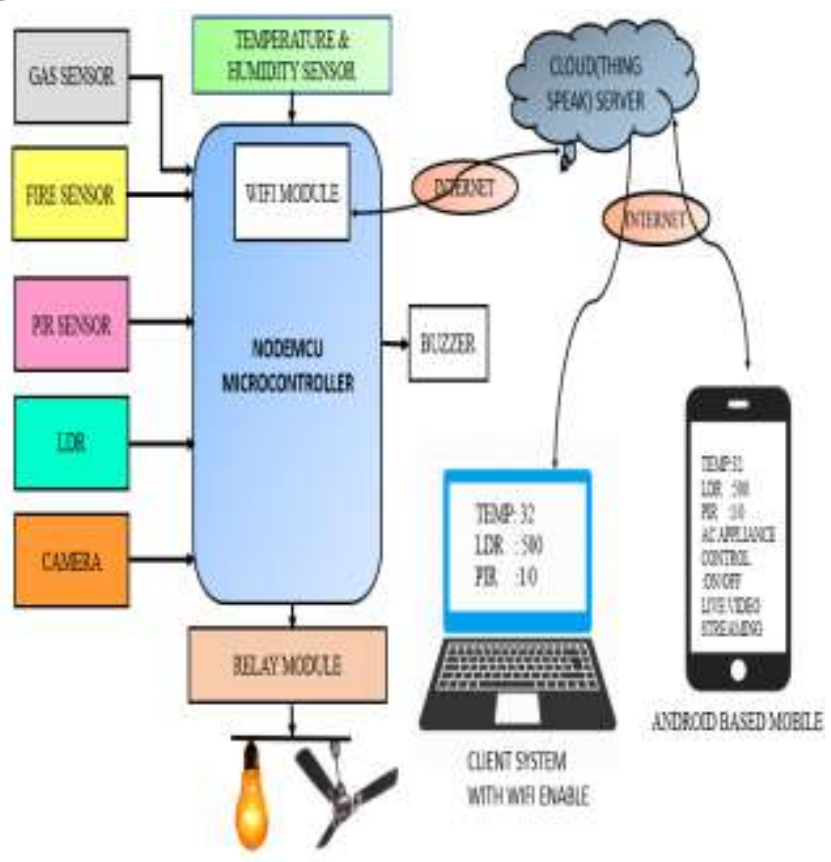

Fig 1: Block diagram of IoT based Smart Home Automation and Scrutiny System

The main theme of this system is to monitor and control the home appliances using IoT. For security purpose, we use PIR sensor and camera. If any person enters the house, then a PIR sensor will detect the movements of that person then automatically camera ON. It captures the person image and sends to the web server through NodeMCU. Using IoT platform, Android apps we can monitor the sensor parameters from anywhere in the world with the help of Internet facility. If sensor readings are abnormal and entered person is unauthorized then we can control some appliances (fan, bulb, AC, buzzer) and sends an alert to the user using IoT concept.

III NODEMCU: The NodeMCU is a WiFi board based on the ESP-12E module. NodeMCU is the open source Internet of Things (IoT) based board.. It fuses firmware which is continuously runs on a ESP8266 Wi-Fi SoC from the Espressif frameworks, and equipment which relies upon the ESP-12 module. The "NodeMCU" is referred to as firmware which is opposed to the advancement pack. It enables you to program the ESP8266 WiFi module with the simple Arduino IDE.

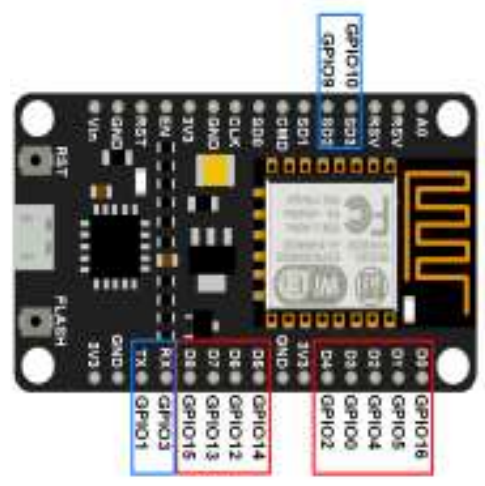

Fig 2: Node MCU

\section{DHT11:}

DHT11 is a Humidity and Temperature Sensor. It consists of 3 main components. A resistive type humidity sensor, an NTC (negative temperature coefficient) thermistor (to measure the temperature) and an 8-bit microcontroller, which converts the analog signals from both the sensors and sends out single digital signal. This digital signal can be read by any microcontroller or microprocessor for further analysis.

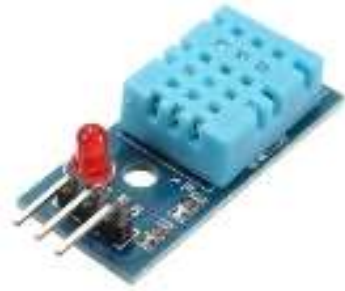

Fig 3. DHT11 Sensor

Interfaciag DHTII with NODE MCC

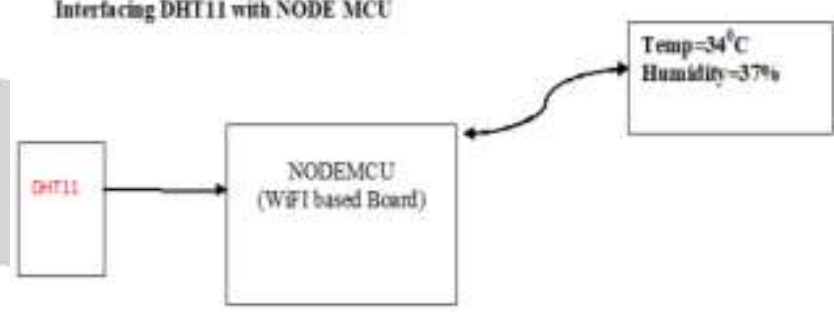

Fig 4. Interfacing DHT11 Sensor with NODE MCU

dht library is available in arduino IDE to read the digital data from DHT11 and to separate the temperature and humidity data

\#include<dht.h>

dht mydht;

dht library function calls are

mydht.read11(pin no).. it reads the data from required pin number

mydht.temperature it produces temperature value mydht.humidity it produces humidity value.

\section{LDR( LIGHT-DEPENDENT RESISTOR )}

An LDR is a component that has a (variable) resistance that changes with the light intensity that falls upon it. This allows them to be used in light sensing circuits. A lightdependent resistor (LDR) is a light-controlled variable resistor. The resistance of this decreases with increasing incident light intensity; in other words, it exhibits photoconductivity. An LDR can be applied in lightsensitive detector circuits, and light- and dark-activated switching circuits.

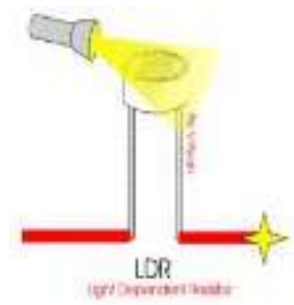

Fig 5. LDR SENSOR 


\section{PIR SENSOR}

PIR is defined as the Passive Infrared Radiation sensor. The passive expression show that the sensor does not effectively participate all the while, which means, it doesn't emanate the alluded IR signals itself, rather inactively recognizes the infrared radiations coming from the human body in the encompassing zone.

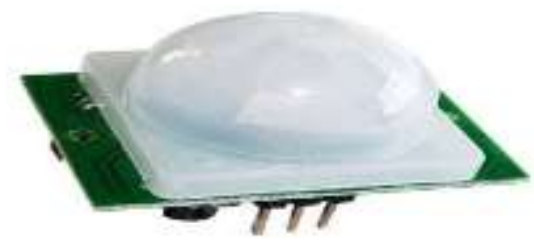

Fig 6. PIR SENSOR

The PIR Sensor is used to detect the motion of the human beings and objects.

Vcc and GND pins of PIR Sensor is connected to the $3.3 \mathrm{~V}$ and ground pins of NodeMCU and the data pin of PIR sensor is connected to the digital pin (D5) of microcontroller.

\section{GAS SENSOR(MQ-2):}

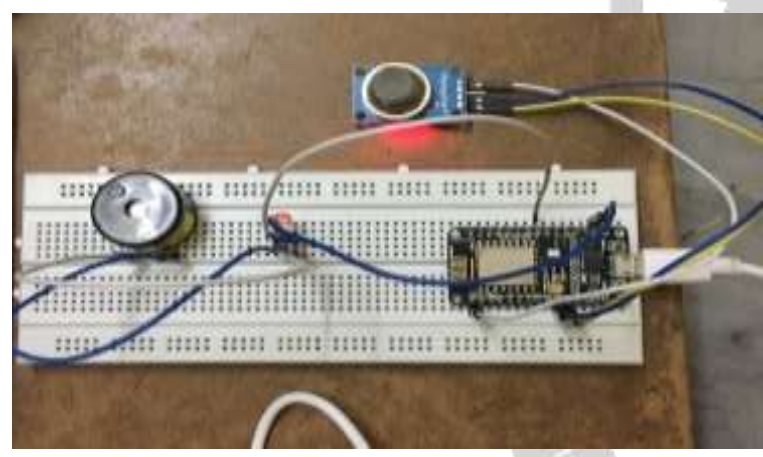

Fig 7. Interfacing MQ2 Sensor with MCU

It detects the gas like Liquified Petroleum Gases (LPG), methane, alcohol and smoke and gives the alert when it increases the Threshold value.Vcc and GND pins of MQ-2 gas sensor is connected to the $3.3 \mathrm{~V}$ and ground pins of NodeMCU. Aout pin of gas sensor is connected to analog pin $\mathrm{A} 0$ of Microcontroller.

\section{FIRE DETECTION SENSOR}

Fire Sensors, Smoke Sensors, Fire Alarms and so forth are a piece of a security hardware that help us in keeping our homes, workplaces and stores safe from flame mishaps. Practically all modern houses, condos, shopping centers, film lobbies, theaters, places of business and shops are furnished with such security devices and it is compulsory in certain areas to fire safety gadgets.

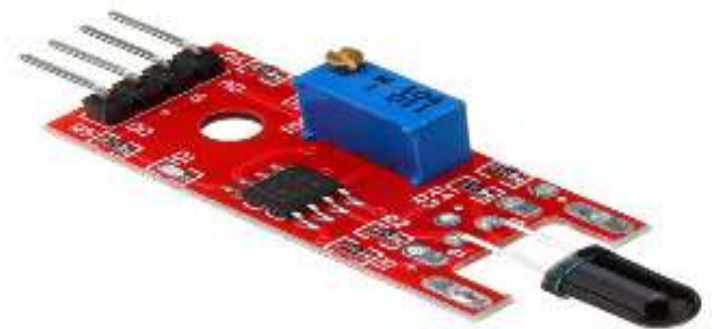

Fig 8 Fire Detection Sensor (KY-026 Sensor)

In this Arduino sketch we'll read values from both digital and analog interfaces on the KY-026, use a ligther or a candle to interact with the flame detector module.

The digital interface will send a HIGH signal when fire is detected by the sensor, turning on the LED on the Node MCU.

\section{CAMERA MODULE}

The Arducam camera module is prepared for taking full HD 1080p photo and video and can be controlled naturally. Arducam camera is the latest version of ArduCAM shield. It is having high definitions SPI camera and it reduce the camera controls the interface complexity. It can be utilized in different platforms as long as they have I2C and SPI interfaces like Raspberry Pi, Arduino, Chipkit, Maple, Beaglebone black modules. It has low cost and provides capability to add different cameras to a one microcontroller.

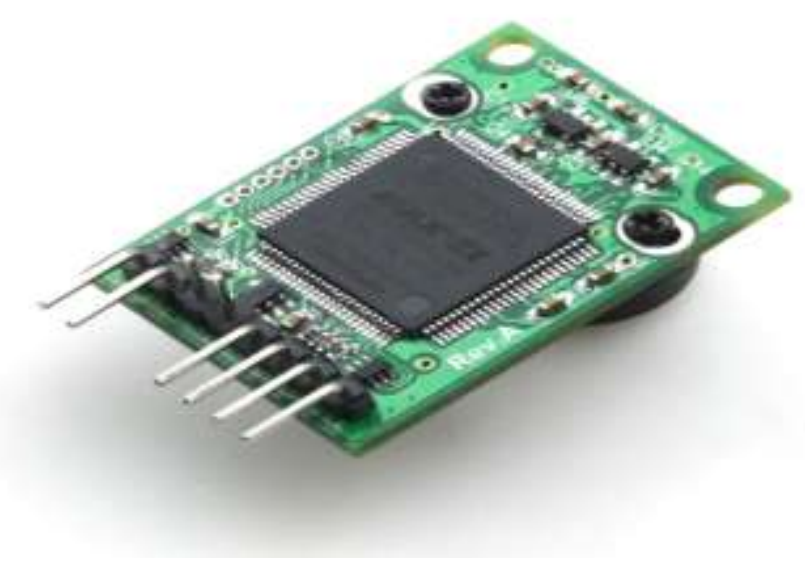

Fig 9: Camera module

\section{ARDUINO IDE:}

Arduino is an open source tool for making computers that can sense and control more of the physical world than your desktop computer. It's an open-source physical computing platform based on a simple micro-controller board, and a development environment for writing software for the board. Arduino can be used to develop interactive objects, taking inputs from a variety of switches or sensors, and controlling a variety of lights, motors, and other physical outputs. 


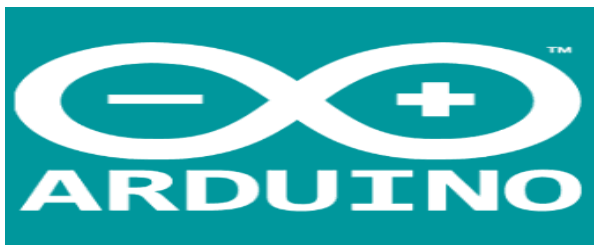

Fig 10: Arduino IDE logo

\section{THINGSPEAK:}

ThingSpeak is an open source IoT (Internet of Things) platform. Using this user can collect the data from cloud and store the sensors data in the cloud and develop IoT applications.Thing speak apps are used for Analyze and visualize stored data in MATLAB and act on data.
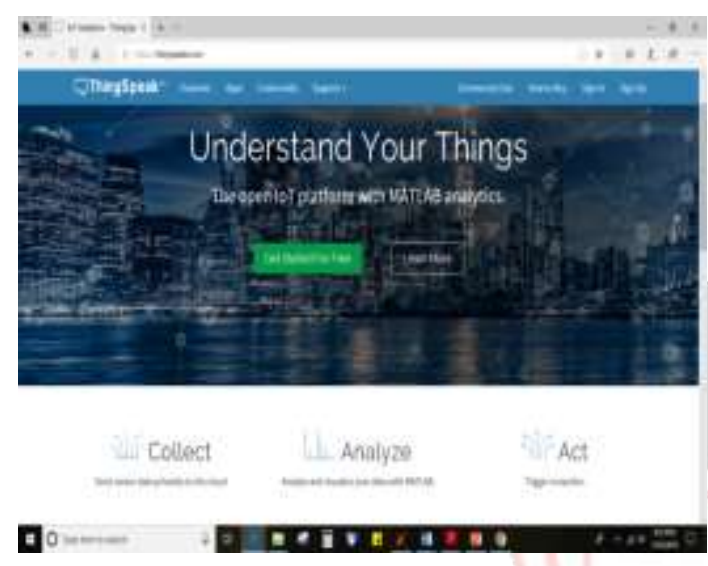

Fig 11.ThingSpeak platform Window

\section{FLOWCHART}

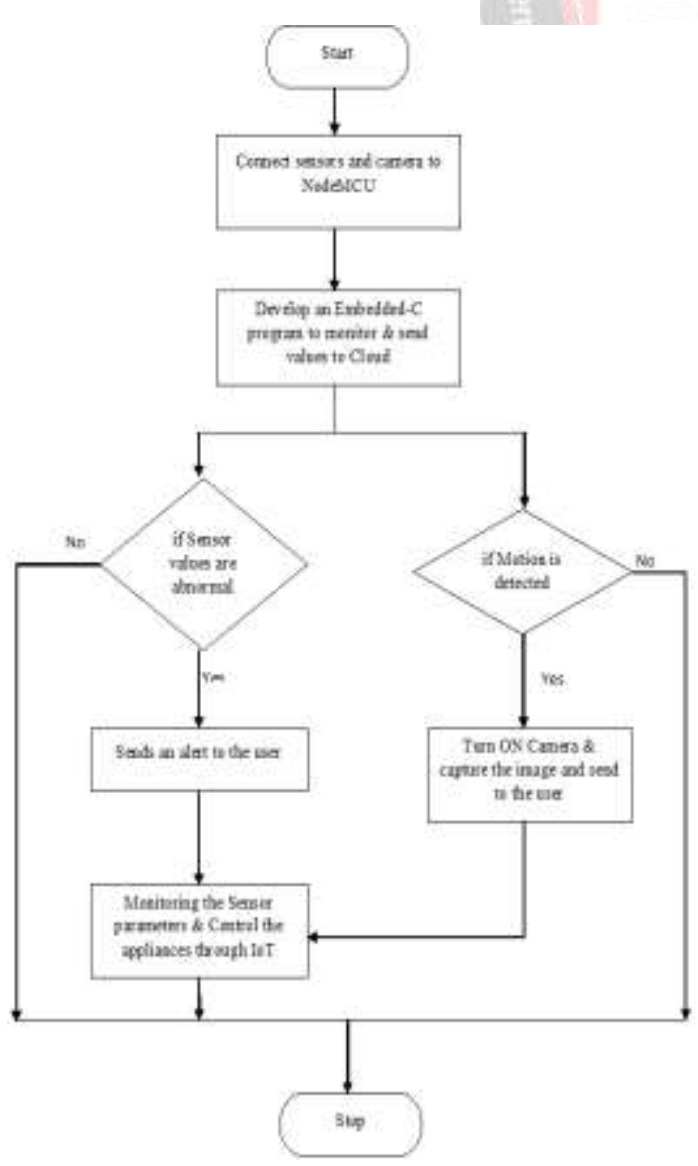

Fig 12: Flow Chart of the Proposed System

\section{IMPLEMENTATION}

The main theme of this System is to monitor and control the home appliances using IoT. Using IoT platform, Android apps we can monitor the sensor parameters from anywhere in the world with the help of Internet facility. If sensor readings are abnormal or entered person is unauthorized then the system sends an alert to the user Through email or twitter using IoT concept and Turn ON the Buzzer when fire is detected or gas leakage. We monitor the sensors reading and we can also control some appliances (such as fan, bulb, AC, buzzer).

In this implementation model we used NODE MCU board,Sensors and Actuators as an Embedded device for sensing and storing the data in to cloud(Thing speak).

Sensors are connected to the Node MCU board. Its read the sensors and on chip ADC will convert the corresponding sensor reading to its digital value and from that values the corresponding environmental parameter will be evaluated.

Here we are developed " Embedded C "code in Arduino IDE to read the sensors data, process sensors data,sends Data to cloud and receive commands from cloud to control the appliances.

Arduino IDE is an open-source electronics platform based on easy-to-use hardware and software.

\section{Advantages of Arduino IDE}

Open source, Easy to develop the applications with the availability of predefined functions. It support all (editor, compiler, debugger, linker and loader .etc) the tools in a single IDE. It supports many libraries (like WiFi, GSM, LCD, ETHERNET, RTOS, SPI, I2C, DHT, ONEWIRE and ESP8266 etc) for interfacing devices. Easy to add the board packages from board manager.

Table 1 Arduino IDE Predefined functions set

\begin{tabular}{|c|c|}
\hline Predefined function & Description \\
\hline pinMode(pin, INPUT OUTPUT) & To specify particular pin as input or cutput \\
\hline diefalalWrite(pin value) & Tow rite logic HIGH or LOW rahe to the particular pin. \\
\hline digitaRead, pin) & $\begin{array}{l}\text { To Read the value from a specified digital pin with the } \\
\text { resalt either HIGH or LOW. }\end{array}$ \\
\hline analogRead(pin) & $\begin{array}{l}\text { To Read the value from a specified analog pin wifh a } 10 \text { - } \\
\text { bit resolution. }\end{array}$ \\
\hline analog Write(pin,valve) & To write analog value' to specified PWM pins. \\
\hline delayims) & $\begin{array}{l}\text { Toproduoe some delay in milliseconds. } \\
\text { Ex.delay (1000); wait one second }\end{array}$ \\
\hline $\begin{array}{l}\text { Serial begin(rate) } \\
\text { Serial printin(data) }\end{array}$ & $\begin{array}{l}\text { To initialize the serial oummunication with specified } \\
\text { bend rate. Usually bud mate is } 9600 \\
\text { Toprint dasa on serial monitor, after that cursor will } \\
\text { more to next line }\end{array}$ \\
\hline Serial read() & Io read fhe cata from serial port. \\
\hline Serial available? & $\begin{array}{l}\text { To check whether data is available or not in Serial } \\
\text { buffer. }\end{array}$ \\
\hline $\begin{array}{l}\text { Serial readBytedintil(character, } \\
\text { buffer length) }\end{array}$ & $\begin{array}{l}\text { Toread data from serial port until it receives a specific } \\
\text { chancter }\end{array}$ \\
\hline
\end{tabular}


DHT11 sensor is connected to one of the digital pin of Node MCU,LDR circuit arrangement is connected to Analog pin (A0) of NodeMCU.

\section{Temperature \& Humidity Calculation:}

Relative Humidity = (density of water vapor / density of water vapor at saturation) $\mathrm{x} 100 \%$.The DHT11 sensor is easy to connect to the Node MCU. we need to install the DHT11 library. It contains following functions.

- DHT.read11(Pinno);

- DHT.Temprature;

- $\quad$ DHT.Humidity ;

By calling these functions we will obtain the temperature and humidity values.

DHT11 sensor gives current Temperature and Humidity values of the room and these sensor readings are sent to the cloud and retrieving these sensor readings from cloud using ThingView or Blynk server app. Depending on the values of the sensor, we can control the appliances from anywhere.

Temperature $=31$ Degrees centigrades

Humidty $=30 \%$.

\section{LIGHT INTENSITY CALULATION:}

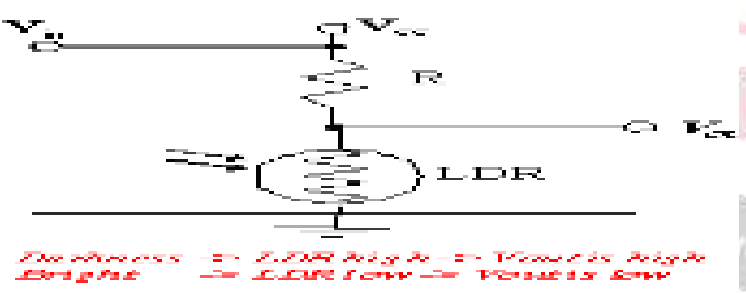

Fig 13. LDR circuit arrangement with NODE MCU.

Output across LDR is given to analog pin of Node MCU.

When light is falling on LDR its resistance decreases then voltage across its decreases, ADC reading decreases.

When no light is falling on LDR its resistance increases then voltage across its increase, ADC reading also increases.

By calling analogRead(A0) we will get Analog to digital conversion value.

$\mathrm{ADC}$ reading=analogRead $(\mathrm{A} 0)$;

Voltage $=$ ADC reading $* 5 /(1023)$;

ADC Reading $=400$ to 650 when brightness

ADC Reading $=700$ to 1023 when darkness

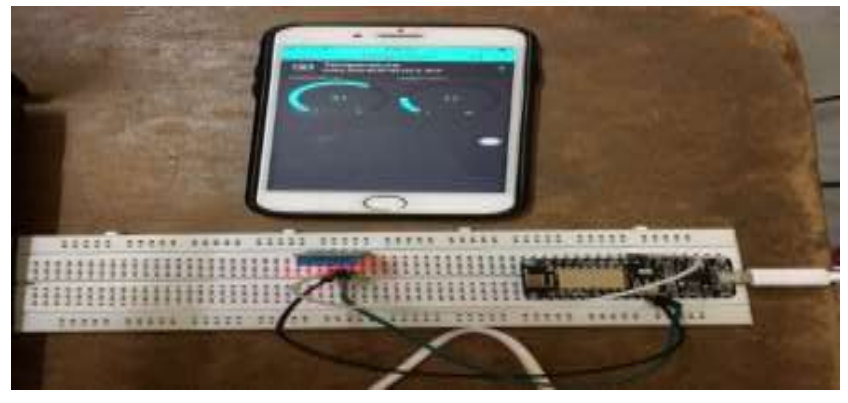

Fig 14. Interfacing DHT11 sensor with NODE MCU.

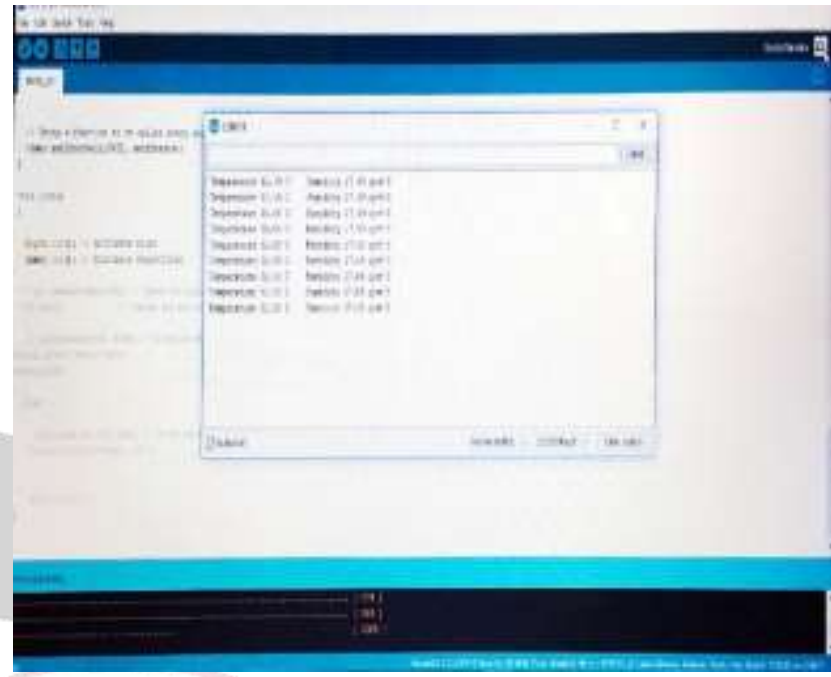

Fig 15. Observing the Temperature and Humidity values on Serial monitor

When the person moves infront of the sensor then the PIR sensor detects the motion of person and also make a camera module will be on, buzzer sound and led light will glows as a indication. If there is no persons near the sensor, the buzzer sound and led light glow will be in OFF. These can be viewed in client system also as shown in figure below

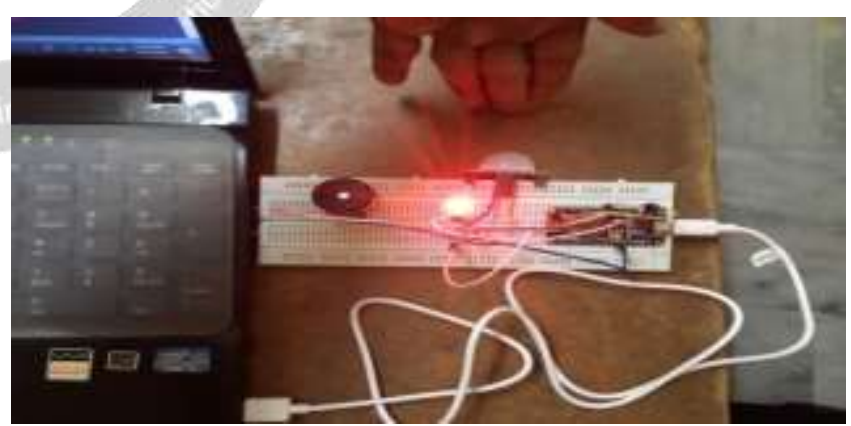

Fig 16. Interfacing PIR sensor with NODE MCU.

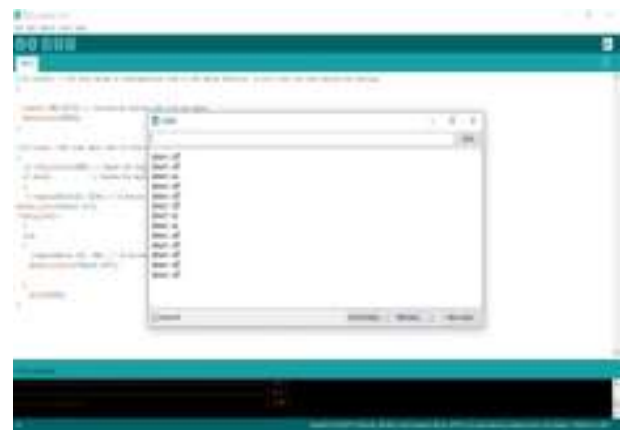

Fig 17 Observing the PIR sensor values on Serial monitor 
Gas sensor:Vec and GND pins of MQ-2 gas sensor is connected to the $3.3 \mathrm{~V}$ and ground pins of NodeMCU. Aout pin of gas sensor is connected to analog pin A0 of Microcontroller.

It detects the gas (LPG) and smoke and gives the alert when it increases the Threshold value.

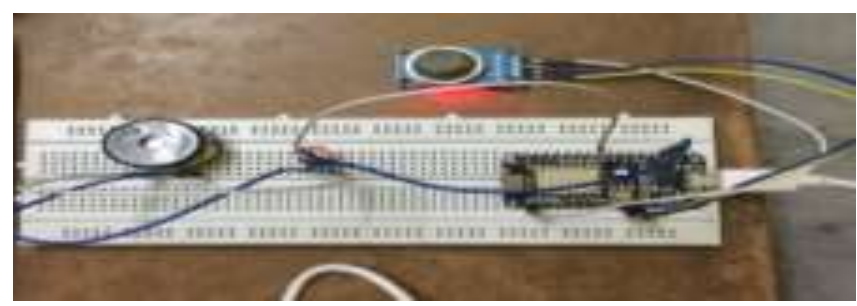

Fig 18 Interfacing gas sensaor with MCU.

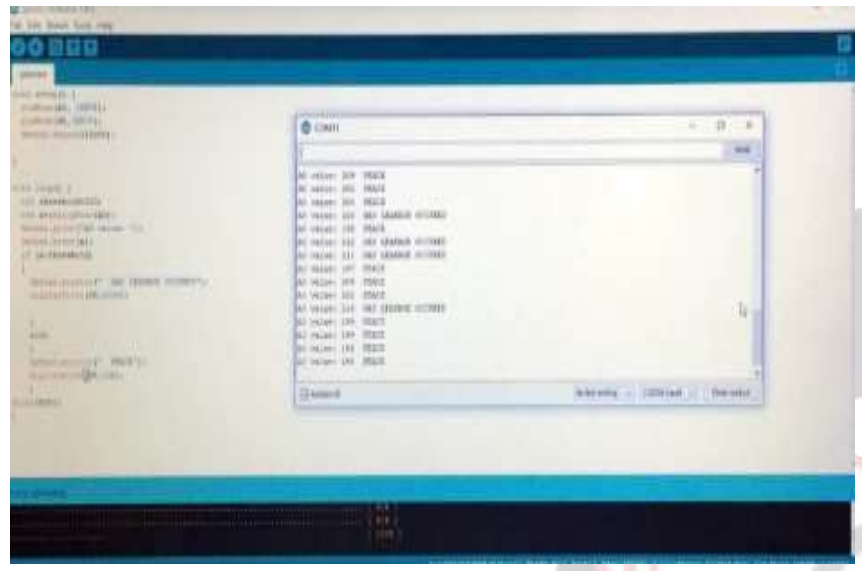

Fig 19 Observing the gas sensor values on Serial monitor

Fire Sensor: It detects the fire, when a fire is put near to this sensor it will gives the indication to the mobile while sending a mail through wifi module.

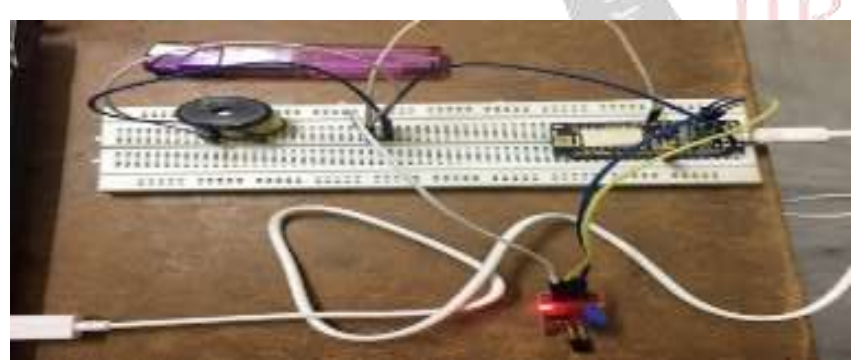

Fig 20 Interfacing fire sensor with Node MCU

\section{Procedure To Send Sensors Data To Cloud}

Connect all the sensors and Actuators to the NodeMCU. NodeMCU process the sensors data and sends the sensors reading to the cloud i.e; ThingSpeak server. Here we are developed Embedded C code to read the sensors data , process sensors data, sends Data to cloud and receive commands from cloud to control the appliances.

ThingSpeak can be used with our IoT module to provide a continuous connectivity with our device from internet.

In order to access the thing speak platform first we create an account in ThingSpeak.com. After successfully creation of an account in thing speak we can create a channel in ThingSpeak.
Each ThingSpeak channel has write and read API keys those can be used to store and retrieve data remotely. ThingSpeak channel is similar to database field. it can be used to store sensor data.Each channel can store maximum of 8 fields of data.

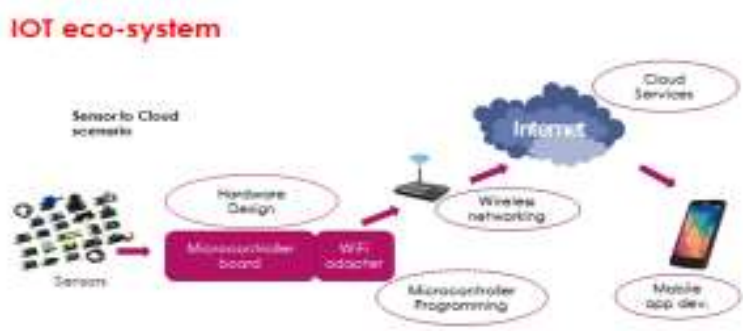

Fig 21 IoT based Eco System for sensors to cloud scenario

We are using Thingspeak.h library to send sensors data to Thing speak server.

\section{Thingspeak.h library functions are}

Thingspeak.begin(client)..it is used to initialize the Thingspeak library.

ThingSpeak.writeField(ChannelNumber,Field1, data, myWriteAPIKey). It is used to send the sensors data to Thingspeak server,

ThingSpeak.readIntField(ChannelNumber,1,

myReadAPIKey). It is used to receive commands/Instructions from cloud to control the appliances.

\section{Program To Push The Sensors Data To Cloud(ThingSpeak).}

\#include <ESP8266WiFi.h>

\#include <WiFiClient.h>

\#include <ThingSpeak.h>

const char* ssid = "IoT"; //Your Network SSID

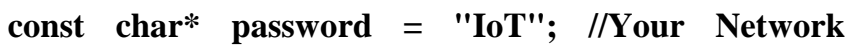
Password

WiFiClient client;

unsigned long myChannelNumber =688413; //Your Channel Number (Without Brackets)

const char *myWriteAPIKey= "EJ4CX0K6ICJE5KKK"; //Your Write API Key

void $\operatorname{setup}()$

\{

Serial.begin(9600);

delay(10);

// Connect to WiFi network

WiFi.begin(ssid, password);

ThingSpeak.begin(client);

\}//setup close

void $\operatorname{loop}()$ 
\{

int a=analogRead(A0);

Serial.print("Idr ADC VALUES:");

Serial.println(a);

ThingSpeak. writeField(myChannelNumber, 1,a,

myWriteAPIKey); //Update in ThingSpeak

$\operatorname{delay}(15000) ;\}$

Data stored in the ThingSpeak web service can be retrieved from the android app on the mobile phone.

ThingView app is used to see the sensor data stored in the ThingSpeak in graphical format.

Easy way of visualise Thing speak channel by using Thingview app.Retrieving those readings in ThingView app and in client system if any values are abnormal ThingSpeak server sends an alert to authorized person Through Twitter or email .

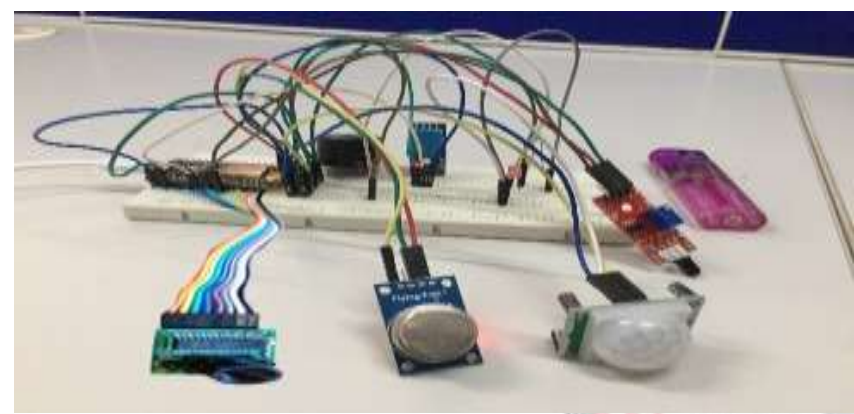

Fig 22 Interfacing all sensors and actuators with NodeMCU

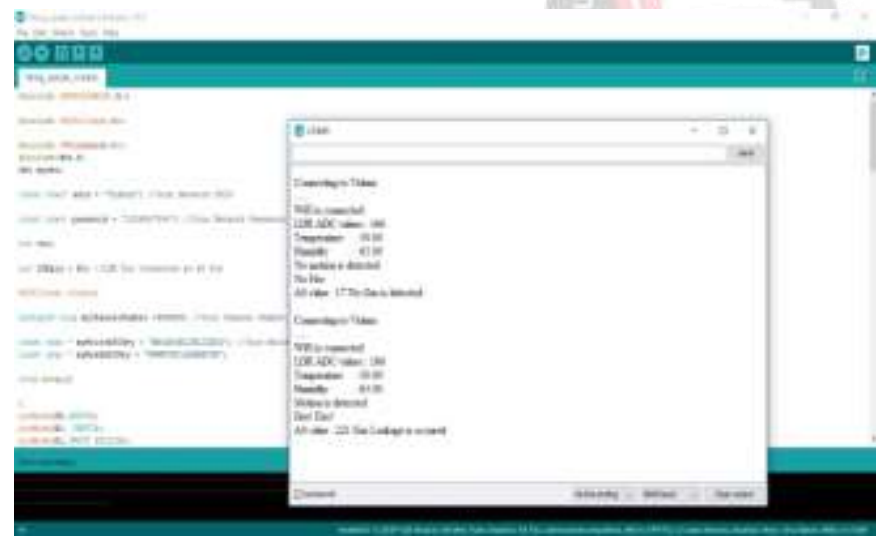

Fig 23 All sensors reading on serial monitor

RESULT:

LDR ADC VALUE $=650$

Temperature $=31$ Degrees centigrades

Humidity $=73$

Gas leakae detected

PIR sensor $=1$ motion detected

Camera on

Fire $=0$ no fire detected

Mobile apps are used to monitor the sensors reading. if any sensor values are abnormal we can control the appliances at the field using Android or IOS apps with the help of IoT platforms.

Procedure to send commands from cloud to actuators

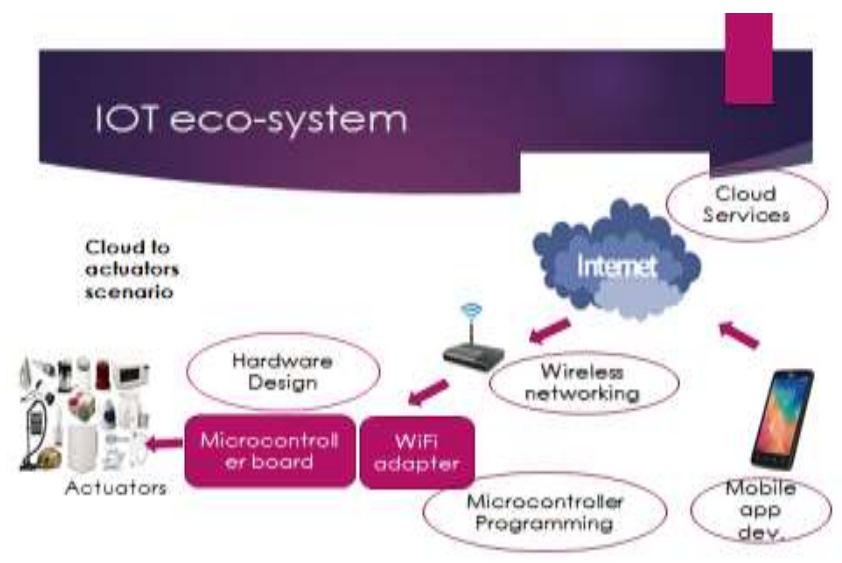

Fig 24 IoT based Eco System for cloud to actuators scenario.

ThingSpeak.readIntField(ChannelNumber,1, myReadAPIKey). Function is used to receive commands/Instructions from cloud to control the appliances.

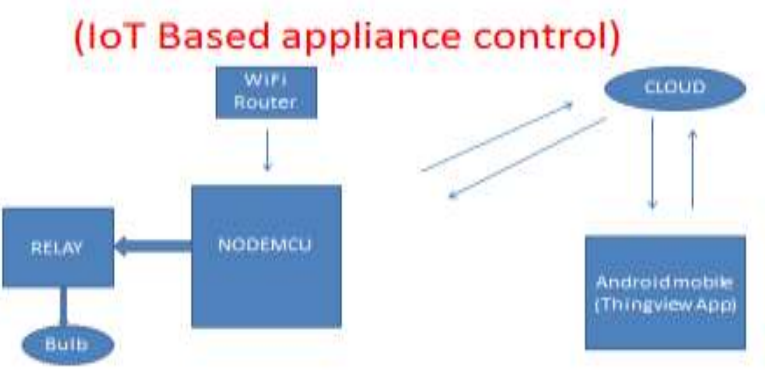

Fig 25 IoT based appliance control system.

Program to IoT Based appliance control system.

\#include "ThingSpeak.h"

\#include <ESP8266WiFi.h>

char ssid[] = "IoT"; // your network SSID (name)

char pass[] = "IoT"; // your network password

WiFiClient client;

unsigned long myChannelNumber $=688413$; / modify this with your own Channel Number

const char * myReadAPIKey = "4H9KSBOHLJDGCD7I"; void $\operatorname{setup}()$

\{

Serial.begin(9600); WiFi.begin(ssid, pass);

ThingSpeak.begin(client);

pinMode(D5, OUTPUT);//relay is connected D5 pin of Node MCU 
\}

void $\operatorname{loop}()\{$

// Read the latest value from field 2 of your channel Int a;

$\mathrm{a}=$ ThingSpeak.readIntField(myChannelNumber, 1 , myReadAPIKey);

Serial.print("readValue = ");

Serial.println(a);

if $(\mathrm{a}==1)\{$ digitalWrite(D0, LOW); else

\{

digitalWrite(D0, HIGH); \}

\}

Here ThingSpeak app is used to send the commands from Mobile to ThingSpeak server or URL link is used to send the commands to ThingSpeak server. Then IoT based Smart Home Automation and Scrutiny System control the appliances based on the received commands from ThingSpeak server(cloud).

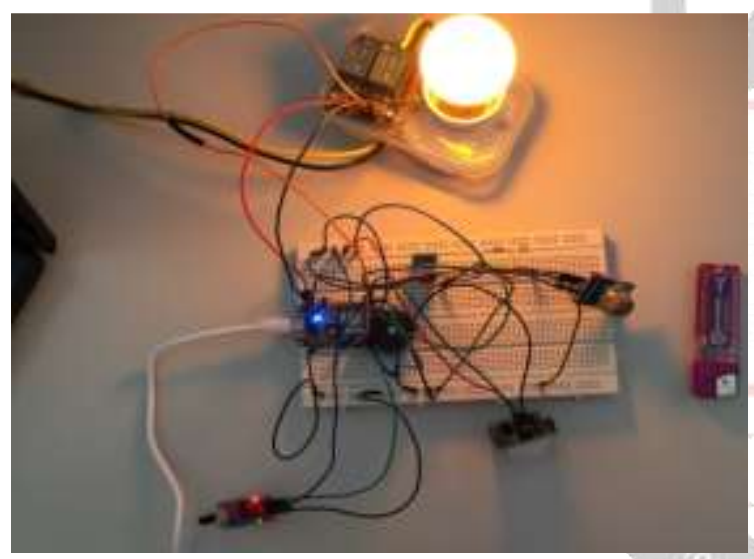

Fig 26 IoT based appliance control system.
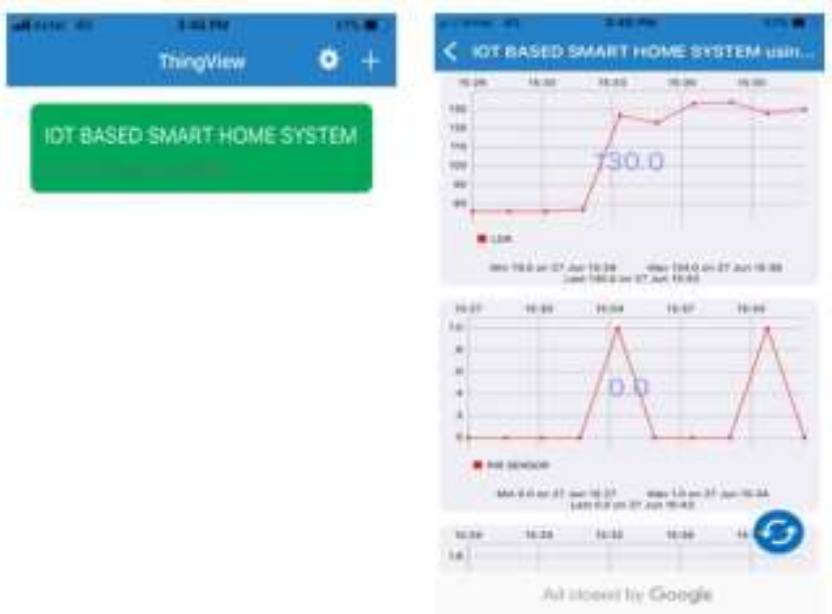

Fig 27 Graphical representation of Sensor values in ThingView app.

These all sensor readings are automatically sent to the cloud server i.e; ThingSpeak server and we can observe those readings at anytime www.thingspeak.com/channels/808503/private_show or using thing view app.

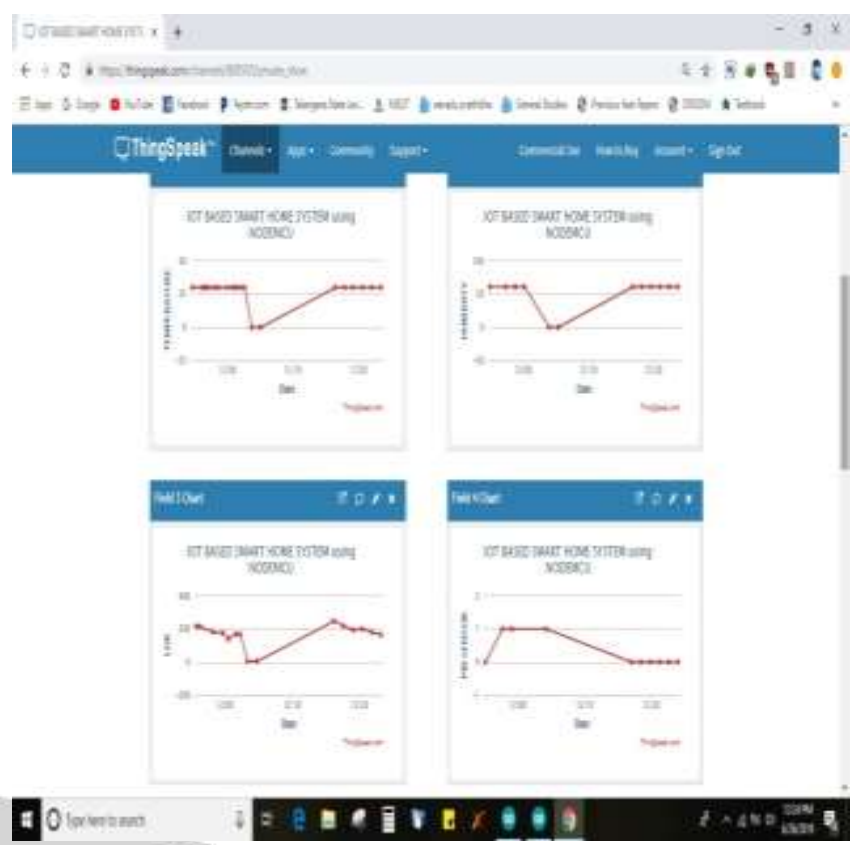

Fig 28 Graphical representation of Sensor values in ThingSpeak channel

ThingView is a mobile application which is used to monitor and analyze of graphs of sensor readings.If the sensor readings are abnormal, then the authorized person will get a notification or alert message via user twitter account as shown in figure below.

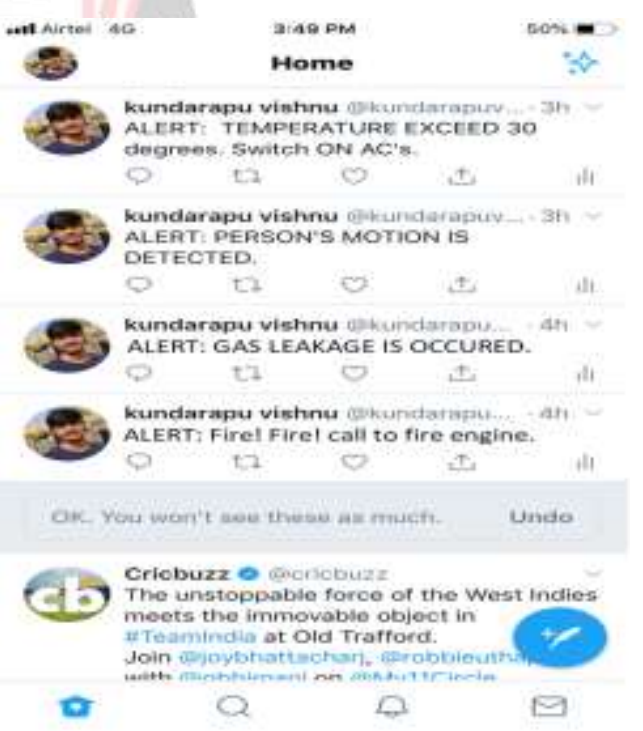

Fig 29 Alert message in Twitter account

\section{CONCLUSION}

This system is appropriate for continuous home monitoring, controlling the AC appliances and providing security (capturing the images) to the home using IoT. Monitoring the parameters like humidity, Temperature, light intensity, gas, fire, water level, motion and image from anywhere with the help of internet. The smart security system is capable of capturing video/recording/image and transmitting to a smart phone in the presence of the internet. If Data is abnormal then authorized persons to get a 
notification via e-mail. By using android based apps we can monitor and control the home appliances. The system might be utilized in numerous spots like banks, hospitals, labs, workplaces and so on that drastically curtailed the danger of unapproved entry.

Home automation technology seeks to reduce your stress by ensuring your home is secure even when you are far away. It is also meant to reduce the amount of effort you put every day into running your household so you can focus more on yourself and the people inside of it. Imagine if your home could automatically save you money, time and effort. With many of these already established and actively improving systems, these ambitions are possible.

\section{FUTURE SCOPE}

Smart Homes makes use of AI(Artificial Intelligence ) and Internet of Things (IoT) devices such as connected sensors, lights, and meters to collect and analyze data. This data is used in making the effective use of home infrastructure, utilities and more to ease up the everyday life effectively and efficiently.

AI in smart homes can convert raw sensor data from the connected smart device into a design of behavior that is relevant to our daily life. Devices integrated with AI learn the inhabitants' routine and start to predict the experience that suits depends on that. For example, if there is no one in the house, it won't turn on the heater or a fan or lights, and automatically lock the doors.

Artificial intelligence (AI), also becoming a powerful presence in technology, has been swiftly dominating the home automation market. AI allows us to integrate smart solutions into our everyday tasks. Not only is AI creating solutions to common everyday problems, but it also making life simpler for everyone. Its presence in home automation allows us to control our appliances, secure our homes, and even limit our expenses. The ultimate goal of home automation is to limit the need for human involvement With $\mathrm{AI}$, this goal is well within reach.

\section{REFERENCES}

[1] Ray, P. P. "A survey on Internet of Things architectures. Journal of King Saud University-Computer and Information Sciences 2016.

[2] John A. Stankovic Life Fellow, IEEE "Research Directions for the Internet of Things".

[3] Dinkar R Patnaik Patnaikuni "A Comparative Study of Arduino, Raspberry Pi and ESP8266 as IoT Development Board" International Journal of Advanced Research in Computer Science Volume 8, No. 5, May-June 2017.

[4] "A study on development issues over IoT platforms,protocols and operating system "2016 international conference on innovation,Embedded and computing systems(ICIIECS).
[5] Ravi Kishore Kodali and Vishal Jain " IOT based smart security and Home Automation system" International conference on computing, communication and automation (ICCCA 2016)

[6]European Research Cluster on Internet of Things (2014) http://www.internet-of-things-research.eu/.

[7] B. Udaya Kumar, D. S. Murty, Ch. R. Phani Kumar, "Implementation of Low-Cost Ethernet Based Home Security Using Wireless Sensor Network", Journal published at Algorithms Research, March 2013.

[8] Nashwa El-Bendary, Mohamed Mostafa M. Fouad, Rabie A. Ramadan, Soumya Banerjee and Aboul Ella Hassanien, "Smart Environmental Monitoring Using Wireless Sensor Networks",K15146_C025.indd, 2013.

[9]Moghavvemi M. and Tan. S. "A reliable and economically feasible remote sensing system for temperature and relative humidity measurement". Sensors and Actuators. 2005. 181-185.

[10] Y. Hao and J. Foster, "Wireless sensor networks for health monitoring applications," Physiological Meas., vol. 29, no. 11, pp. R27-R56, 2008

\section{BIOGRAPHIES}

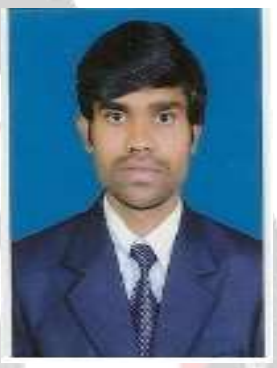

Ome Nerella,

ME, Assistant Professor ECE,GokaRaju Rangaraju Institute of Enginnering and Technology, Nizampet,Hyderabad-500090,

Telangana,India.

Email:omenerella@gmail.com

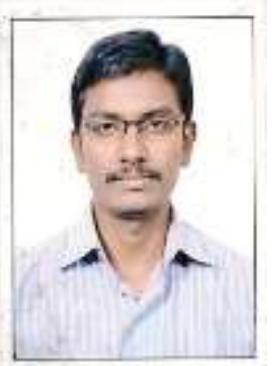

Someswara Rao Gaddala, M.Tech, Assistant Professor in ECEVidya jyothi Institute Of Technology, Aziz Nagar Gate C.B. Post, Hyderabad-500075,Telangana, India. Email:someshg.somu@gmail.com 